Gynäk. Rdsch. 1981;21(Suppl. 2):209-210

\title{
Heterologe Insemination in der freien Praxis des Gynäkologen unter besonderer Berücksichtigung hormoneller Therapie \\ H.W. Horst W. Kupka
}

\section{Düsseldorf}

Dr. Horst W. Kupka, Claudiusstrasse 41, D-4000 Düsseldorf 1 (BRD)

Die Kasuistik umfasst 47 Patientinnen mit 51 Graviditäten im Behandlungszeitraum von Juni 1974 bis November 1979. 21 Konzeptionen (41,18\%) traten schon nach einem

Behandlungszyklus ein. Verwendet wurde bei der heterologen Insemination AID (Artificial Insemination Donor) ausschliesslich Nativsperma an 1-3 aufeinanderfolgenden Tagen mittels Portiokappe nach Fikentscher und Semm. Während der ersten zwei Zyklen traten 31 Konzeptionen (60,78\%) ein. 2 Konzeptionen gelangen erst nach zehn Behandlungszyk-len, 1 nach acht Zyklen. Insgesamt mussten fur die 51 Graviditäten durchschnittlich 2,69 Zyklen inseminiert werden. Drei Graviditäten traten nach vorheriger Hydropertubationslangzeitbehandlung ein.

Von den 51 Graviditäten kamen 22 (43,14\%) unter Hormontherapie zustande. Das Durchschnittsalter betrug hierbei 32,09 Jahre, das Durchschnittsalter der nicht hormonell behandelten Frauen betrug 28,45 Jahre, während das Gesamtdurchschnittsalter aller 51 Graviden 30,02 Jahre betrug. Die jüngste Frau war 24 Jahre alt, die älteste 40 Jahre.

Freie Vorträge II 210 Indikationen zur Hormonbehandlung stellten dar:

Ovulationstiming mit HMG/HCG oder LH-RH, um die Anzahl der Inseminationen pro Zyklus bei weit gereisten Patientinnen zu vermindern

Zyklusanomalien

Zusatztherapie bei mindestens drei erfolglos verlaufenden Behandlungszyklen mit primär anscheinend normalen Ovulationen und Zyklen in der Lutealphase Verbesserung der Zervixfaktoren.

Endometriose mit Infertilität Hyperprolaktinämie mit Infertilität Verteilung der Konzeption unter Hormontherapie

$\%$

836,36

9,09

13,64

31,81

4,55 4,55 4,55 
Unter der Hormontherapie traten 3mal Gemini (alle unter Clomiphenzitrat) ein. 1 Frau abortierte während der Frühgravidität, wurde dann jedoch nach drei Zyklen wieder schwanger. Der Abort ist in den Zahlen nicht enthalten. Geht man von den 64 Frauen aus, von denen 9 die Therapie nach einem erfolglosen Zyklus abbrachen, so stellen die 47 erfolgreich behandelten Frauen einen Anteil von 73,44\% dar. Dariiber hinaus ergibt sich bei den 51 eingetretenen Graviditäten bei 55 Frauen eine Erfolgsrate von sogar 92,73\%. Diese ist teilweise durch die mehrmalige Insemination während eines Zyklus zur Zeit der Ovulation und durch die hormonell beeinflusste Ovulationsauslòsung zu erklären.

Erörterungen über Indikationen, allgemeine Voraussetzungen, Inseminationstechnik, Spenderauswahl oder juristische, theologische oder genetische Aspekte müssen wegen des vorgegebenen engen Rahmens entfallen.

L. Literatur- und Hormonpräparateverzeichnis beim Verfasser. 